\title{
Podocyte foot process broadening in experimental diabetic nephropathy: amelioration with renin-angiotensin blockade
}

\author{
S. A. Mifsud ${ }^{1}$, T.J. Allen ${ }^{2}$, J. F. Bertram ${ }^{3}$, U.L.Hulthen ${ }^{2}$, D.J.Kelly ${ }^{4}$, M.E. Cooper ${ }^{2}$, J.L. Wilkinson-Berka ${ }^{1}$, \\ R. E. Gilbert ${ }^{4}$ \\ ${ }^{1}$ Department of Physiology, University of Melbourne, Victoria, Australia \\ ${ }^{2}$ Department of Medicine, Austin and Repatriation Medical Centre, Victoria, Australia \\ ${ }^{3}$ Department of Anatomy and Cell Biology, Monash University, Victoria, Australia \\ ${ }^{4}$ Department of Medicine, St. Vincent's Hospital, Victoria, Australia
}

\section{Abstract}

Aims/hypothesis. Changes in podocyte number and morphology have been implicated in the pathogenesis of proteinuria and the progression of human and experimental kidney disease. This study sought to examine podocyte foot process and slit pore architecture in experimental diabetic nephropathy and to determine whether such changes were modified with renoprotective intervention by blockade of the renin-angiotensin system.

Methods. The number of filtration slits per $100 \mu \mathrm{m}$ of glomerular basement membrane was assessed by transmission electron microscopy and quantitated histomorphometrically in control animals and in rats with 24 weeks of streptozotocin-induced diabetes. Diabetic rats were either untreated or received the angiotensin converting enzyme inhibitor ramipril, or the angiotensin II type 1 receptor antagonist, valsar$\tan$.

Results. When compared with control animals, diabetes was associated with a decrease in the number of slit pores per unit length of glomerular basement membrane, indicative of podocyte foot process broadening. Both ramipril and valsartan attenuated these ultrastructural changes to a similar degree. These differences remained after correcting for glomerular volume as a possible confounding variable. Conclusion/interpretation. Preservation of podocyte architecture could contribute to the renoprotective effects of renin-angiotensin system blockade in diabetic nephropathy. [Diabetologia (2001) 44: 878-882]

Keywords Podocyte, foot process, diabetic nephropathy, ramipril, valsartan.
The kidney and, in particular, the glomerulus undergo major structural changes in diabetes. Changes in mesangial matrix and glomerular basement membrane (GBM) width in diabetes have been studied extensively where the expansion of the mesangial matrix was found to relate closely to declining glomerular filtration rate [1]. The contribution of the glomerular visceral epithelial cell or podocyte to progressive

Received: 19 January 2001 and in revised form: 28 March 2001

Corresponding author: Dr. Richard Gilbert, Department of Medicine, St. Vincent's Hospital, Fitzroy, Victoria, Australia, 3065. E-mail: gilbert@medstv.unimelb.edu.au

Abbreviations: GBM, Glomerular basement membrane; RAS, renin-angiotensin system; $\mathrm{AT}_{1}$, angiotensin II type 1; SBP, systolic blood pressure; GV, glomerular volume. renal disease has also been evaluated in diabetic and non-diabetic renal disease $[2,3]$. Unlike the mesangial and endothelial cell components of the glomerulus, the podocyte is terminally differentiated and largely incapable of replication. In addition to constituting the filtration slit diaphragm, the podocytes, like pericytes elsewhere have a key role in counteracting the expansile forces of glomerular capillary pressure [4].

The most commonly identified change in podocyte structure is foot process effacement in which the interdigitating pattern of podocyte attachment to the GBM undergoes substantial simplification. Such changes are frequently identified in experimental [5-7] and human kidney diseases [8]. Agents which block the renin-angiotensin system (RAS), such as ACE inhibitors have been shown to not only reduce 
Table 1. Physical and biochemical parameters at end of study.

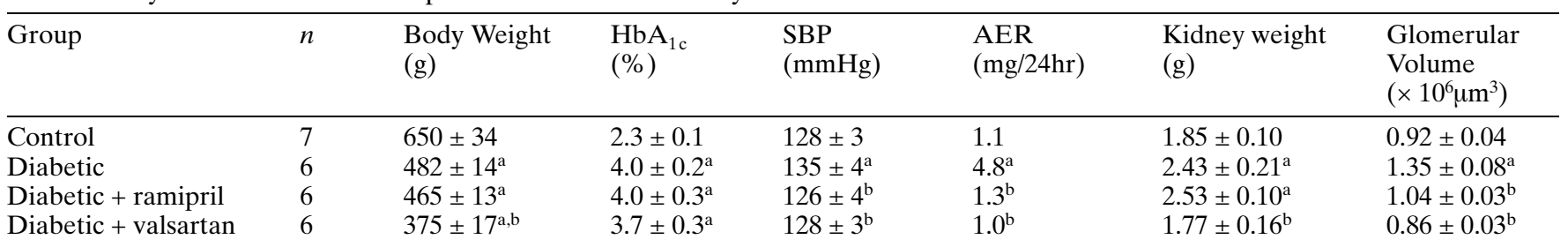

$\mathrm{HbA}_{1 \mathrm{c}}$, glycated haemoglobin; SBP, systolic blood pressure; AER, urinary albumin excretion rate in male Sprague-Dawley rats 24 weeks after induction of control vehicle or diabetes.
Data are expressed as means \pm SEM except AER expressed as geometric mean. ${ }^{\mathrm{a}} p<0.01$ vs control, ${ }^{\mathrm{b}} p<0.01$ vs diabetic. intraglomerular pressure [9] but to also decrease proteinuria and the rate of decline of renal function in diabetic nephropathy [10]. These multiple effects have been attributed to a variety of mechanisms [11] although changes in the glomerular podocyte have not been specifically evaluated, despite the presence of functioning angiotensin II type $1\left(\mathrm{AT}_{1}\right)$ receptors on this cell type [12].

This study thus sought to examine changes in podocyte foot processes in experimental diabetes and to determine the effect of blockade of the RAS on these changes.

\section{Materials and methods}

Male Sprague-Dawley rats (225-250 g) at 8 to 10 weeks of age, were rendered diabetic with streptozotocin $\left(50 \mathrm{mg} / \mathrm{kg}^{-1}\right.$; Boehringer-Mannheim, Mannheim Germany) or received citrate buffer to serve as controls. Diabetic rats were then randomized to receive one of the following regimens: (1) no treatment; (2) the ACE inhibitor ramipril $1 \mathrm{mg} / \mathrm{kg}$ in drinking water (Hoechst, Frankfurt, Germany) or (3) the $\mathrm{AT}_{1}$ receptor antagonist valsartan $30 \mathrm{mg} \cdot \mathrm{kg}^{-1} \cdot \mathrm{day}^{-1}$ by gavage (Novartis, Basel, Switzerland). Only diabetic animals with plasma glucose concentrations greater than $15 \mathrm{mmol} / \mathrm{l}$ were included in the study. Diabetic animals were maintained on a dose of 4 units of long-acting insulin (Ultralente; Novonordisk, Bagsvaerd, Denmark), administered daily to promote weight gain and prevent ketoacidosis. Rats had free access to standard rat chow and drinking water. Experimental procedures adhered to the guidelines of the National Health and Medical Research Council of Australia's Code for the Care and Use of Animals for Scientific Purposes.

After 24 weeks of diabetes, body weight was measured and systolic blood pressure (SBP) measured by tail-cuff plethysmography in conscious pre-warmed rats. Glycated haemoglobin was estimated by high performance liquid chromatography (Biorad, Richmond, Calif., USA) [13]. Rats were placed in metabolic cages (Iffa Credo, L'Arbresele, France) for $24 \mathrm{~h}$ to determine albuminuria by radio-immunoassay [14].

Glomerular Structure. Transmission electron microscopic morphometric analysis was carried out to accurately quantify changes in glomerular structure [15]. Renal histology was assessed by quantitative histomorphometry [16]. Animals were anaesthetised with pentobarbital sodium and the kidneys were perfused in vivo at arterial pressure via an intra-aortic cannula with saline, followed by $2.5 \%$ glutaraldehyde. Kidney sections were then prepared for light (paraffin sections) and electron microscopic examination. The glomerular cross-sectional area (GA) was measured in 30 glomerular profiles per rat, using a videoimaging system (Video Pro 32, Leading Edge, Bedford Park, South Australia, Australia) connected to a Zeiss AXIOPHOT microscope (Oberkocken, Germany). The glomerular volume $(\mathrm{GV})$ was then calculated as: $\mathrm{GV}=(\beta / \mathrm{k})(\mathrm{GA})^{3 / 2}$, where $\beta=1.38$ is the size-distribution coefficient and $\mathrm{k}=1.1$ is the shape coefficient for glomeruli idealized as a sphere [17]. For electron microscopy, twenty evenly spaced electron micrographs were obtained at a final magnification of $\times 18000$ from one randomly chosen thin section from one randomly selected glomerulus from each rat. To determine the number of filtration slits per $100 \mu \mathrm{m}$ of GBM, the number of filtration slits was obtained by counting the number of slit pores from each set of micrographs. The length of the GBM was calibrated and then measured using the computer-based program Sigma Scan Pro 4.0. All measurements were done in a masked fashion.

Statistical Analysis. All data are shown as means \pm SEM unless otherwise specified. Because of a positively skewed distribution albuminuria was logarithmically transformed before analysis and shown as geometric means. Data were analysed by analysis of variance with comparisons between group means by Fisher's least significant difference method. A $p$ value of less than 0.05 was considered as statistically significant.

\section{Results}

Metabolic and Biochemical Parameters. After 6 months, diabetes was associated with reduced body weight gain compared to control rats $(p<0.01)$ and was not improved with either ramipril or valsartan treatment (Table 1). Diabetes was associated with an increase in glycated haemoglobin, SBP, AER and kidney weight (Table $1 ; p<0.01$ ). In rats treated with either ramipril or valsartan, SBP and AER were similar to that in control animals. Glycated haemoglobin was not affected by any of the drug regimens.

Glomerular Structure. Control rats showed a thin GBM with relatively evenly spread filtration slit pores (Fig.1A). In contrast, diabetes was associated with a diminution in the number of slit pores per $100 \mu \mathrm{m}$ of GBM $(p<0.01)$ indicative of foot process 


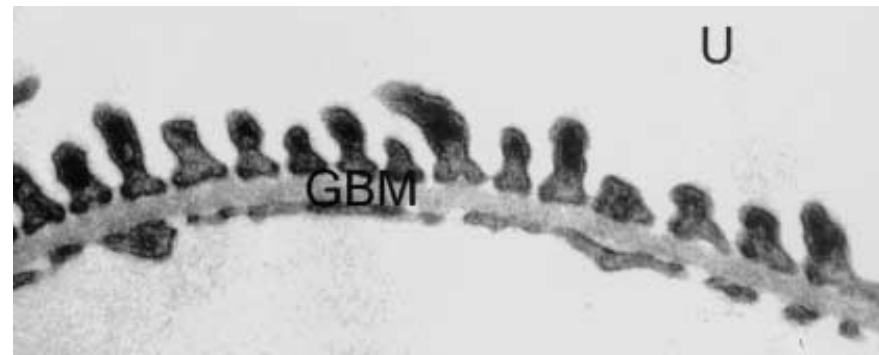

CAP

A

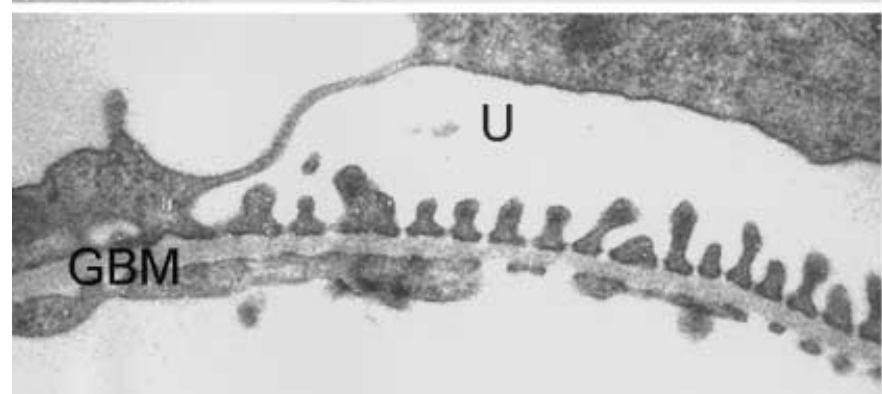

CAP

C

Fig. 1A-D. Transmission electron micrographs of representative glomerular capillary loops from Sprague-Dawley rats 24 weeks after induction of control vehicle or diabetes. Control (A), diabetic (B), diabetic + ramipril (C) and diabetic + valsartan (D). GBM, glomerular basement membrane; CAP, capillary lumen; U, urinary space (Magnification $\times 14200$ )

broadening (Fig. 2A). In addition, irregularity in podocyte foot process dimensions and spacing were also noted along with thickening of the GBM (Fig.1B). These effects were attenuated by treatment with both ramipril (Fig. 1C), and valsartan (Fig.1D). These quantitative changes persisted in all groups after correcting for glomerular volume (Table 1), as a possible confounding variable (Fig. 2B).

\section{Discussion}

Experimental diabetes was associated with a reduction in the number of slit pores per unit length of GBM foot indicative of foot process broadening. In both ramipril- and valsartan-treated diabetic rats slit pore number approximated that of control animals. These findings suggest that not only is podocyte foot process structure altered in experimental diabetes but that it can be attenuated by agents which interrupt the RAS.

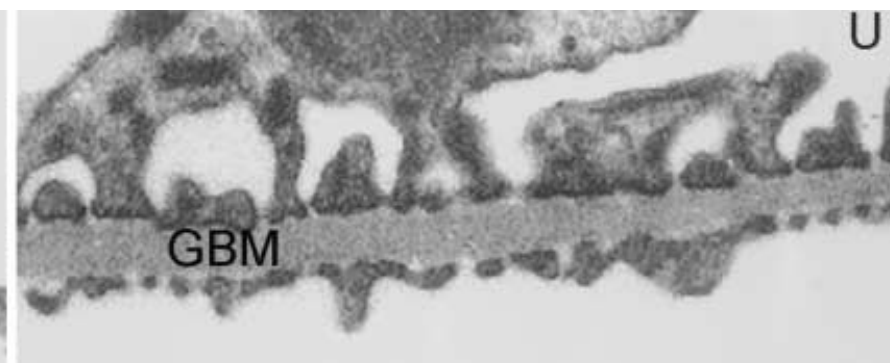

\section{CAP}

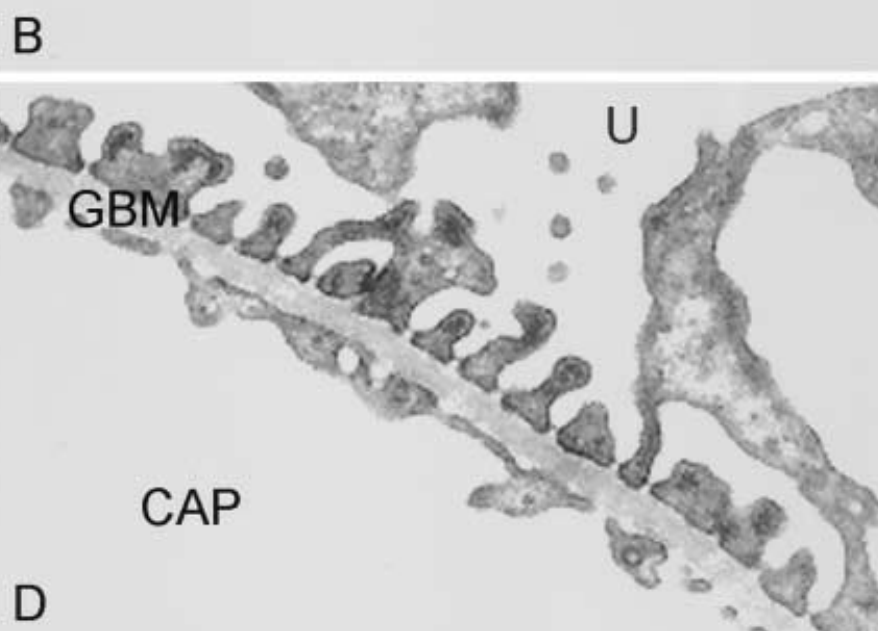

In humans, podocyte loss develops with progressive albuminuria, such that in patients with overt nephropathy the total number of podocytes per glomerulus is reduced, without change in glomerular surface area [3]. Therefore, when compared with the glomeruli of control subjects, in diabetic nephropathy each podocyte is obliged to cover a greater surface area. The reduction in slit pore number per length of GBM as found in our study suggests that not only can podocytes not replicate but that remaining podocytes are not able to elaborate a sufficient number of foot processes to compensate for this loss. We found ramipril and valsartan treatment prevented the reduction in slit number per GBM length in diabetic animals. Whether this reflected a prevention of diabetes-associated podocyte loss or occurred as a consequence of de novo foot process elaboration cannot be determined from our study. However, the recent finding of an anti-apoptotic effect of RAS blockade in diabetes suggests that the former mechanism could be operational [18]. Podocyte number predicts progression of nephropathy in microalbuminuric Pima Indians with Type II (non-insulin-dependent) diabetes mellitus [19], suggesting that prevention of podocyte loss would be therapeutically relevant. Recent studies, however, also suggest a more direct effect of the RAS on the slit pore, showing that angiotensin II induces biochemical modifications in zonula occludens- 1 that are attenuated by ACE inhibitor therapy [20]. A wide range of receptors for vasoactive hor- 

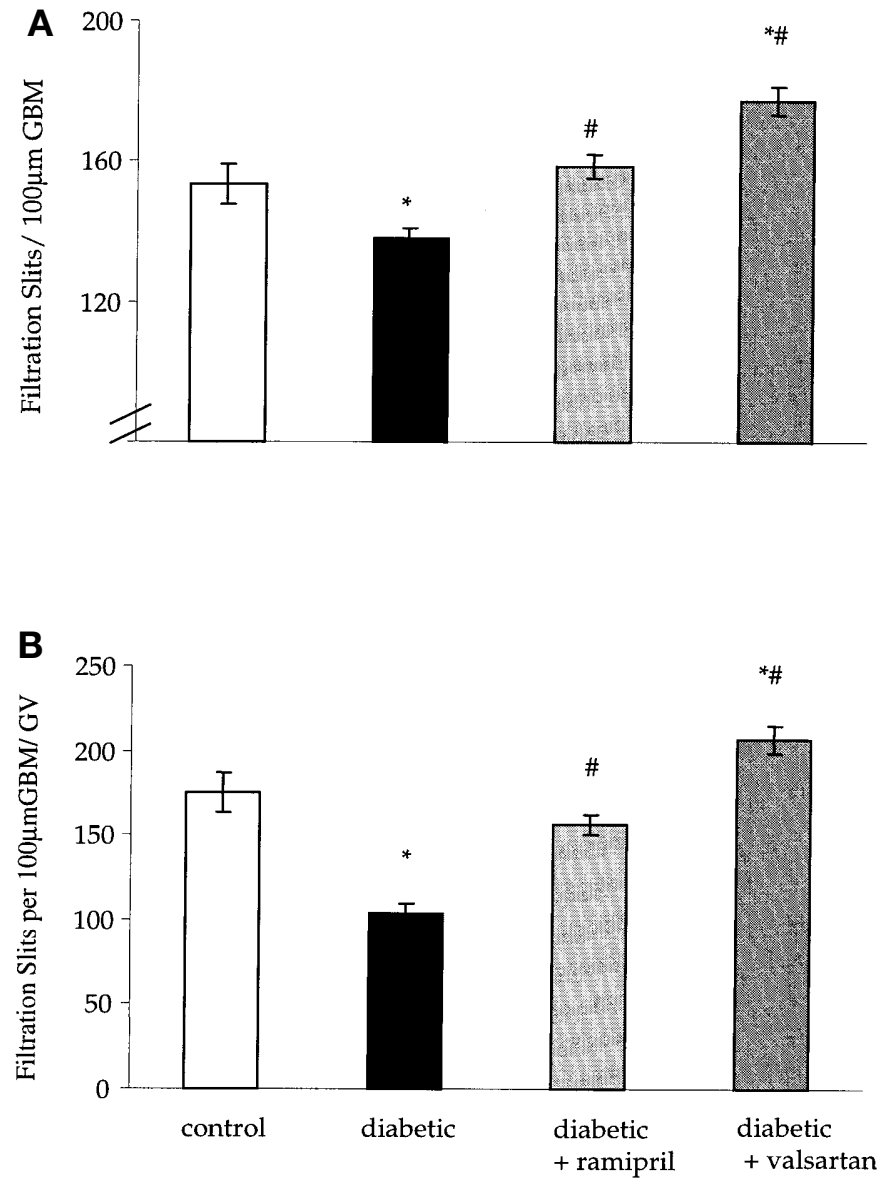

Fig. 2 A, B. The number of filtration slits per $100 \mu \mathrm{m}$ of glomerular basement membrane (GBM) (A), and when the same experimental data are corrected for glomerular volume $(\mathrm{GV})$ (B). Results are shown as means \pm SEM. ${ }^{*} p<0.01$ vs control. \# $p<0.01$ vs diabetic

mones and growth factors have been identified on the podocyte, including the presence of type 1 and type 2 angiotensin II receptors $[12,21]$ and receptors for bradykinin [22].

The relation between glomerular slit pores, proteinuria and glomerular filtration are complex. A reduction in slit pore number per unit capillary length in combination with GBM thickening, as shown in this study, would theoretically both lead to reduced ultrafiltration per capillary surface area [23]. However, these effects are counterbalanced by the increased glomerular volume and increased intraglomerular pressure such that in the presence of a normal ultrafiltration co-efficient $[24,25]$ glomerular hyperfiltration persists. Both ramipril and valsartan have multiple effects on these parameters such that not only did slit pore number approximate those in control animals but similar beneficial effects have also been noted in GBM thickness, glomerular volume and mesangial expansion [26]. These findings suggest that in diabetes, changes in glomerular filtration and protein permeability and the effects of RAS blockade are likely to reflect interactions between epithelial, mesangial and capillary endothelial components of the glomerulus.

We found SBP was increased in diabetic rats and normalised with ramipril therapy. Whereas hypertension is an important factor in the progression of renal disease it has been clearly established in both clinical and experimental studies that agents which block the RAS have a renoprotective effect beyond that due to blood pressure reduction alone $[10,27,28]$. In a model of experimental renal disease, ramipril, but not nifedipine preserved podocyte structure, despite equivalent blood pressure reduction [29] suggesting a specific role for the RAS in influencing podocyte structure in renal diseases.

In summary, experimental diabetes was associated with significant $(p<0.01)$ changes in podocyte foot processes and slit pore number that were attenuated by $\mathrm{ACE}$ inhibition and $\mathrm{AT}_{1}$ receptor blockade. These findings suggest that such structural changes could contribute to altered glomerular function in diabetes and the therapeutic effects of blockade of the RAS.

Acknowledgements. This project was supported by grants from the National Health and Medical Research Council of Australia. R. Gilbert is the recipient of a Career Development Award and D. Kelly is the recipient of a postdoctoral fellowship, both from the Juvenile Diabetes Research Foundation.

\section{References}

1. Mauer SM, Steffes M, Ellis EN, Sutherland DER, Brown DM, Goetz FC (1984) Structural-functional relationships in diabetic nephropathy: J Clin Invest 74: 1143-1155

2. Kriz W, Gretz N, Lemley KV (1998) Progression of glomerular disease: Is the podocyte the culprit? Kidney Int 54: 687-697

3. Pagtalunan ME, Miller PL, Jumping-Eagle S et al. (1997) Podocyte Loss and Progressive Glomerular Injury in Type II Diabetes. J Clin Invest 99: 342-348

4. Kriz W, Hackenthal E, Nobiling R, Sakai T, Elger M, Hahnel B (1994) A role for podocytes to counteract capillary wall distension. Kidney Int 45: 369-376

5. Inokuchi S, Shirato I, Kobayashi N, Koide H, Tomino Y, Sakai T (1996) Re-evaluation of foot process effacement in acute puromycin aminonucleoside nephrosis. Kidney Int 50: 1278-1287

6. Dworkin LD, Hostetter TH, Rennke HG, Brenner BM (1984) Hemodynamic basis for glomerular injury in rats with desoxycorticosterone-salt hypertension. J Clin Invest 73: $1448-1461$

7. Shimamur T, Morrison B (1975) A progressive glomerulosclerosis occurring in partial five-sixths nephrectomized rats. Am J Pathol 79: 95-106

8. Grisham E, Churg J (1975) Focal glomerulosclerosis in nephrotic patients: an electron microscopic study of glomerular podocytes. Kidney Int 7: 111-122

9. Zatz R, Dunn BR, Meyer TW, Anderson S, Rennke HG, Brenner BM (1986) Prevention of diabetic glomerulopathy by pharmacological amelioration of glomerular capillary hypertension. J Clin Invest 77: 1925-1930 
10. Lewis ES, Hunsicker LG, Bain RP, Rohde RD (1993) The effect of angiotensin converting enzyme inhibition on diabetic nephropathy. N Engl J Med 329: 1456-1462

11. Johnston CI, Risvanis J, Naitoh M, Tikkanen I (1998) Mechanism of Progression of Renal Disease - Current Hemodynamic Concepts. J Hypertens 16: S3-S7

12. Gloy J, Henger A, Fischer KG et al. (1998) Angiotensin II Modulates Cellular Functions of Podocytes. Kidney Int 54: S168-S170

13. Allen TJ, Cooper ME, O'Brien RC, Bach LA, Jackson B, Jerums G (1990) Glomerular filtration rate in the streptozotocin diabetic rat: The role of exchangeable sodium, vasoactive hormones and insulin therapy. Diabetes 38: 1182-1190

14. Soulis-Liparota T, Cooper ME, Dunlop M, Jerums G (1995) The relative roles of advanced glycation, oxidation and aldose reductase inhibition in the development of experimental diabetic nephropathy in the Sprague-Dawley rat. Diabetologia 38: 387-394

15. Nyengaard JR (1999) Stereologic methods and their application in kidney research. J Am Soc Nephrol 10: 1100-1123

16. Ricardo SD, Bertram JF, Ryan GB (1994) Antioxidants protect podocyte foot processes in puromycin aminonucleoside-treated rats. J Am Soc Nephrol 4: 1974-1986

17. Weibel ER (1979) Stereological Methods: Practical Methods for Biological Morphometry. London, Academic Press, pp 162-203

18. Kelly DJ, Wilkinson-Berka J, Cox AJ, Cooper ME, Gilbert RE (2000) Apoptosis in progressive diabetic nephropathy in Ren-2 rats. J Am Soc Nephrol A3399 (Abstract)

19. Meyer TW, Bennett PH, Nelson RG (1999) Podocyte number predicts long-term urinary excretion in Pima Indians with Type II diabetes and microalbuminuria. Diabetologia 42: $1341-1344$

20. Macconi D, Ghilardi M, Bonassi ME et al. (2000) Effect of angiotensin-converting enzyme inhibition on glomerular basement membrane permeability and distribution of zonula occludens-1 in MWF rats. J Am Soc Nephrol 11: 477-489

21. Sharma M, Sharma R, Greene AS, McCarthy ET, Savin VJ (1998) Documentation of angiotensin II receptors in glomerular epithelial cells. Am J Physiol 274: F623-F627

22. Pavenstadt H, Spath M, Fiedler C et al. (1992) Effect of bradykinin on the cytosolic free calcium activity and phosphoinositol turnover in human glomerular epithelial cells. Ren Physiol Biochem 15: 277-288

23. Drumond MC, Deen WM (1994) Structural determinants of glomerular hydraulic permeability. Am J Physiol 266: F1-F12

24. Osterby R, Parving H, Nyberg G et al. (1988) A strong correlation between glomerular filtration rate and filtration surface in diabetic nephropathy. Diabetologia 31: 265-270

25. Hostetter TH, Troy J, Brenner BM (1981) Glomerular hemodynamics in experimental diabetes mellitus. Kidney Int 19: 410-415

26. Allen TJ, Cao ZM, Youssef S, Hulthen UL, Cooper ME (1997) Role of angiotensin II and brakykinin in experimental diabetic nephropathy: Functional and structural studies. Diabetes 46: 1612-1618

27. Kasiske BL, Kalil RS, Ma JZ, Liao M, Keane WF (1993) Effect of antihypertensive therapy on the kidney in patients with diabetes: a meta-regression analysis. Ann Intern Med 118: 129-138

28. Anderson S, Rennke HG, Brenner BM (1986) Therapeutic advantage of converting enzyme inhibitors in arresting progressive renal disease associated with systemic hypertension in the rat. J Clin Invest 77: 1993-2000

29. Amann K, Nichols C, Tornig J et al. (1996) Effect of ramipril, nifedipine, and moxonidine on glomerular morphology and podocyte structure in experimental renal failure. Nephrol Dial Transplant 11: 1003-1016 